\title{
Evolution of symbiosis in the Vibrionaceae: a combined approach using molecules and physiology
}

\author{
Michele K. Nishiguchi and Vinod S. Nair \\ Department of Biology, New Mexico State University, Box 30001, MSC 3AF, Las Cruces, \\ NM 88003-8001, USA
}

Correspondence

Michele K. Nishiguchi

nish@nmsu.edu

\begin{abstract}
The family Vibrionaceae is considered to be one of the most diverse and well-studied groups of bacteria. Here, evolution is assessed within the Vibrionaceae to determine whether multiple origins of eukaryotic associations have occurred within this diverse group of bacteria. Analyses were based on a large molecular dataset, along with a matrix that consisted of 100 biochemical and restriction digest characters. By using direct optimization methods to analyse both datasets individually and in combination, a total-evidence cladogram has been produced, which supports the hypothesis that several important symbionts (both mutualistic and pathogenic) within the Vibrionaceae are not monophyletic. This leads us to consider that symbiosis (and subsequently, associations with Eukarya) has evolved multiple times within the Vibrionaceae lineage.
\end{abstract}

\section{INTRODUCTION}

The evolution of microbial pathogens from mutualistic and free-living bacteria has been a targeted area of interest for determining the roles of virulence and specificity among closely related species. Previous studies have used either biochemical or molecular data to elucidate relationships among selected groups of taxa, but none have clarified the transition between free-living and symbiotic species (Bang et al., 1981; Baumann et al., 1983; Nearhos \& Fuerst, 1987). The methods by which pathogens evolve among various clades of micro-organisms and whether particular virulence factors are transferred and then regulated within and between species are common themes that underlie the evolution of pathogenesis. The Vibrionaceae are a group of highly divergent bacteria, which contains both free-living and symbiotic species (Nishiguchi \& Jones, 2003). Members of this family are globally important due to their prevalence in numerous ecological niches. Many species of the Vibrionaceae possess the ability to bioluminesce via the lux operon, although its biological role in bacterial cells remains unknown (Czyż et al., 2000). Many of these luminescent species form symbiotic relationships, both pathogenic and cooperative, with marine organisms. They are found increasingly to be the cause of diseases in many secondary hosts, which consume primary marine hosts that contain

Published online ahead of print on 6 June 2003 as DOI 10.1099/ ijs.0.02792-0.

Abbreviation: TBR, tree bisection and reconnection.

A table showing all biochemical and restriction digest data that were used in the analysis is available as supplementary material in IJSEM Online. concentrated numbers of vibrios (Ruby \& Lee, 1998). Recently, it has been determined that specific virulence factors in pathogenic Vibrio species are exhibited in the colonization of eukaryotic tissue by non-pathogenic Vibrio fischeri (Reich \& Schoolnik, 1994, 1996; Reich et al., 1997; Ruby, 1999b; Stabb \& Ruby, 2003) and may even be required for colonization (Nyholm et al., 2000; Stabb et al., 2001). To colonize an animal host, both pathogenic and mutualistic bacteria must conquer defence mechanisms of the host that discourage the growth of unacceptable micro-organisms. These defences may include environmental conditions within the host, active immune responses and general host defence (Koropatnick et al., 2001; Stewart et al., 2001). As a result, there is a growing number of similarities between the tissue colonization of benign species, such as $V$. fischeri, and that of pathogenic vibrios, such as Vibrio cholerae and Vibrio parahaemolyticus (Makino et al., 2003). Due to these similarities, it has been suggested that many of the characteristics of host-tissue colonization in pathogenic and benign species may have arisen from a common ancestral origin (McFall-Ngai, 1999; Ruby, 1999a).

In this study, we have attempted to complete a phylogenetic survey of the Vibrionaceae by using DNA sequence data from three molecular loci [16S rRNA, the intergenic region of the lux operon (luxRI) and glyceraldehyde phosphate dehydrogenase $(g a p A)]$ and 100 non-sequence characters (biochemical and restriction digest patterns), in order to determine which evolutionary patterns are prevalent and influential for radiation among this species-rich family of $\gamma$-Proteobacteria. Total sequence data comprise approximately $3.6 \mathrm{~kb}$ per complete strain. These loci were selected due to their ability to resolve some of the internal clades 
within the Vibrionaceae (gapA and luxRI), as well as to distinguish among distantly related clades (16S rRNA). This combination of molecular loci should delineate phylogenetic history at various hierarchical levels; thus, the resolution of the analyses should distinguish among the span of species (by using 16S rRNA) and strains (by using gapA and luxRI). With the knowledge that colonization of host species is a particularly important aspect of the lifehistory strategy of some species of the Vibrionaceae, we combined the molecular and morphological attributes of 75 representative biotypes to determine whether there was an ancestral split among different symbiotic biotypes of Vibrionaceae, leading to several species that have evolved the ability to associate with a specific eukaryotic host.

\section{METHODS}

Isolation of bacteria. Bacterial isolates were obtained from squid or fish light organs by previously described methods (Nishiguchi et al., 1998; Nishiguchi, 2000, 2002). Briefly, bacterial strains were isolated from loliginid, sepiolid or monocentrid light organs, or were obtained from various laboratories (Table 1). Light organs were dissected out of the eukaryotic host and homogenized in sterile sea water, then subsequently diluted and plated on Luria-Bertani high-salt (LBS) [32 parts per thousand (p.p.t.)] agar plates (Ruby \& Asato, 1993). Colonies were isolated the next day and grown in $5 \mathrm{ml}$ LBS medium overnight at their optimum temperature $\left(18-28^{\circ} \mathrm{C}\right)$ and frozen as stocks until they were utilized. Squid and fish symbionts were identified according to previously described methods (Nishiguchi et al., 1998; Nishiguchi, 2000).

Biochemical analyses of isolates, utilization of carbon type and RFLP scoring. Bacterial strains from frozen or fresh stock were cultured on LBS agar plates overnight at their optimum temperatures $\left(18-28^{\circ} \mathrm{C}\right)$. Single c.f.u. of each strain were obtained the following day and liquid cultures were grown to an $\mathrm{OD}_{600}$ of 0.5 in $10 \mathrm{ml}$ LBS medium. The culture was then centrifuged in $50 \mathrm{ml}$ Sorvall tubes at 5000 r.p.m. for $10 \mathrm{~min}$ in a refrigerated centrifuge $\left(5{ }^{\circ} \mathrm{C}\right)$. The pellet was resuspended gently in $5 \mathrm{ml} \mathrm{PBS}(\mathrm{pH} 7 \cdot 3)$ and centrifuged at 5000 r.p.m. for $10 \mathrm{~min}$. This step was repeated to ascertain complete removal of LBS medium. The pellet was then

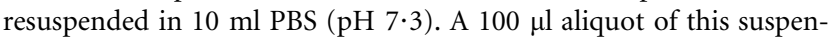
sion was added to each of the 96 wells of a GN2 Biolog plate and incubated overnight at their optimum temperature $\left(18-28^{\circ} \mathrm{C}\right)$. Plates were scored colorimetrically for the carbon source present in each well (see Supplementary Table in IJSEM Online). Each Biolog plate was completed in duplicate to ensure reproducibility of the results. Biochemical phenotypes were recorded in a data matrix by using MacClade 4.05 (Maddison \& Maddison, 2002), with a score of 0 for no utilization and 1 for utilization of each substrate in the Biolog plate. In total, 95 carbon sources were measured (see Supplementary Table in IJSEM Online). Here we treated each phenotypic feature, as measured by the Biolog plate, as our primary homology statement sensu de Pinna (1991). It is phylogenetic analysis that decides ultimately whether the primary homology statement survives the homology test (secondary homology). For RFLPs, we scored the number of fragments per restriction digest from previously reported data (Urakawa et al., 1999). The fragments comprised the last five of the 100 characters used in the biochemical/ RFLP data matrix. These characters were scored by the number of fragments produced by each restriction enzyme (i.e. if an enzyme produced four fragments from one strain, the score in the matrix for that RFLP would be 4).
Isolation, amplification and sequencing of bacterial loci. Isolation of DNA was completed by initially growing pure cultures of each strain in $5 \mathrm{ml}$ LBS broth overnight. A sample of each culture $(2 \mathrm{ml})$ was centrifuged for $1 \mathrm{~min}$ and the medium was removed. DNA from each culture was then isolated by using a DNeasy isolation kit (Qiagen). Once purified DNA was obtained, 1-10 ng template DNA was used for PCR amplifications. For the 16S rRNA locus, four sets of primers (each amplifying approximately $420 \mathrm{bp}$ ) were used to complete the entire $16 \mathrm{~S}$ rRNA gene sequence ( $1600 \mathrm{bp})$. These consisted of: $1 \mathrm{~F}$ (5'-AGAGTTTGATCMTGGCTCAG-3') and 4R (5'-AGGCCTTCTTCATACACGCG-3'); 2F (5'-GCAAGCCTGATGCAGCCATG-3') and 3R (5'-ATCGTTTACGGCGTGGACTA- $\left.3^{\prime}\right)$; 3F (5'-AAACAGGATTAGATACCCTG-3') and 2R (5'-CTGGTCGTAAGGGCCATGAT-3'); and 4F (5'-AGGTGGGGATGACGTCAAGT- $\left.3^{\prime}\right)$ and 1R (5'- AAGGAGGTGWTCCARCC- $\left.3^{\prime}\right)$. 16S rRNA gene amplification consisted of one cycle at $94{ }^{\circ} \mathrm{C}$ for $2 \mathrm{~min}$, followed by 25 cycles of the following: $94{ }^{\circ} \mathrm{C}$ for $15 \mathrm{~s}, 49^{\circ} \mathrm{C}$ for $15 \mathrm{~s}$ and $72^{\circ} \mathrm{C}$ for $15 \mathrm{~s}$. Reactions were terminated by holding samples at $72{ }^{\circ} \mathrm{C}$ for $7 \mathrm{~min}$. For the luxRI locus, primers used to amplify the fragment were LuxRIF ( $5^{\prime}$-CAGCGGTTAGTTGTATTGAG-3') and LuxRIR (5'-AGCAAAACGRCTTAATTC-3'), which amplified a gene product of approximately $1200 \mathrm{bp}$. luxRI amplification consisted of one cycle at $94^{\circ} \mathrm{C}$ for $2 \mathrm{~min}$, followed by 25 cycles of the following: $94^{\circ} \mathrm{C}$ for $15 \mathrm{~s}$, annealing for $15 \mathrm{~s}$ at temperatures from 42 to $49^{\circ} \mathrm{C}$ and extension at $72{ }^{\circ} \mathrm{C}$ for $30 \mathrm{~s}$. The reaction was terminated by holding samples at $72^{\circ} \mathrm{C}$ for $7 \mathrm{~min}$ after amplification was completed. For the gapA locus, primers were gapAF (5'-GGATTTGGCCGCATCGGCCG-3') and gapAR (5'-CCGAACTCGTTGTCGTACCA-3'), which amplified a gene product of $\sim 900 \mathrm{bp}$. Samples were amplified by using the following procedure: one cycle at $94^{\circ} \mathrm{C}$ for $2 \mathrm{~min}$, followed by 25 cycles of $94{ }^{\circ} \mathrm{C}$ for $15 \mathrm{~s}, 36-38^{\circ} \mathrm{C}$ for $30-40 \mathrm{~s}$ and $72{ }^{\circ} \mathrm{C}$ for $30 \mathrm{~s}$. The reactions were terminated by holding samples at $72{ }^{\circ} \mathrm{C}$ for $7 \mathrm{~min}$. Two internal primers were used for each of the luxRI and gapA loci, to obtain complete double-stranded sequences. These were: gapAFInt (5'-CCTGAATTGCTYGGWSAACA-3') and gapARInt (5'-CACCGAGATGCGTTAACAATG-3'); and LuxRIFInt (5'-GAATGAGGAGACTACCTTAC-3') and LuxRIRInt (5'-GCCGTAAATACAGAAAGTTTG-3'). All PCRs had similar concentrations of magnesium $(2.5 \mathrm{mM})$, dNTPs $(200 \mu \mathrm{M}$ each; Promega), primers $(0.2 \mu \mathrm{M}$ of each forward and reverse primer), $1 \times$ reaction buffer ( $10 \mathrm{mM}$ Tris/ $\mathrm{HCl}, \mathrm{pH} 9 \cdot 0,50 \mathrm{mM} \mathrm{KCl}, 0 \cdot 1 \%$ Triton X-100) and $0 \cdot 2 \mathrm{U}$ Taq polymerase (Promega) or AmpliTaq (Applied Biosystems). Once all PCR templates were amplified, reactions were cleaned with a GENECLEAN II DNA purification kit (BIO 101). All samples were pre-sequenced by using Applied Biosystems BigDye (version 3.1) and excess fluorescently labelled dNTPs were removed by spin column or plate (Edge Biosystems). Samples were sequenced on an Applied Biosystems 3100 automated capillary sequencer. Doublestranded sequences were combined and checked for chromatograph errors by using the program Sequencher (version 4.0; Gene Codes). All luxRI and gapA sequences and approximately $80 \%$ of the $16 \mathrm{~S}$ rRNA loci were sequenced in our laboratory. 16S rRNA gene sequences were also used to confirm the identity of light organ isolates by comparing the full sequence to those in the Ribosomal Database Project (http://rdp.cme.msu.edu/html).

Phylogenetic analysis. Sequence data were analysed by using the direct optimization method described by Wheeler (1996) and implemented in the computer program POY (Wheeler et al., 2002). This method directly assesses the number of DNA sequence transformations (evolutionary events) required by a phylogenetic topology, without the use of multiple sequence alignment. This is accomplished by generalization of existing character optimization procedures, including insertion and deletion events (indels) in addition to base substitutions. The crux of the method is the treatment of indels as processes, as opposed to the patterns implied by multiple 
Table 1. List of all Vibrionaceae strains used in the phylogenetic analyses, host species or culture collection accession number (if applicable), genes sequenced for each strain and GenBank accession numbers for these sequences

\begin{tabular}{|c|c|c|c|c|c|}
\hline Strain & Source & Location & 16S rRNA & gapA & $\operatorname{luxRI}$ \\
\hline Listonella pelagia & ATCC $25916^{\mathrm{T}}$ & & X74722 & & \\
\hline Photobacterium damselae & ATCC $33539^{\mathrm{T}}$ & & X74700 & & \\
\hline Photobacterium phosphoreum & Anomalopid fishes & & AY292916 & & AY292963 \\
\hline Photobacterium leiognathi RM1 & Rondeletiola minor & France (Banyuls sur Mer) & AY292947 & & AY292983 \\
\hline Photobacterium leiognathi SN2B & Sepiolina nipponensis & Japan (Tosa Bay) & AY292951 & & \\
\hline Psychrobacter sp. EH201 & Euprymna hyllebergi & Thailand (Rayong) & AY292940 & & \\
\hline Vibrio aerogenes & FG1, CCRC $17041^{\mathrm{T}}$ & & AF124055 & & \\
\hline Vibrio aestuarianus & ATCC $35048^{\mathrm{T}}$ & & X74689 & & \\
\hline Vibrio carchariae/Vibrio harveyi & Paralichthys dentatus & & AF134581 & & \\
\hline Vibrio carchariae/Vibrio harveyi EH701 & Euprymna hyllebergi & Thailand (Rayong) & AY292941 & & \\
\hline Vibrio cholerae & Environmental isolate & & AY292952 & & AY292964 \\
\hline Vibrio cincinnatiensis & ATCC $35912^{\mathrm{T}}$ & & X74698 & & \\
\hline Vibrio diabolicus & $\mathrm{HE} 800^{\mathrm{T}}$ & & X99762 & & \\
\hline Vibrio diazotrophicus & ATCC $33466^{\mathrm{T}}$ & & X56577 & & \\
\hline Vibrio fischeri & ATCC $7744^{\mathrm{T}}$ & & AY292938 & & AY292977 \\
\hline Vibrio fischeri CG101 & Cleidopus gloriamaris & Australia (Townsville) & AY292939 & AY292958 & AY292978 \\
\hline Vibrio fischeri EB12 & Euprymna berryi & Japan (Tosa Bay) & AY292921 & AY292954 & AY292968 \\
\hline Vibrio fischeri EM17 & Euprymna morsei & Japan (Tokyo Bay) & AY292922 & AF034846 & AY292969 \\
\hline Vibrio fischeri ES114 & Euprymna scolopes & Hawaii, USA (Kaneohe Bay) & AY292919 & AF034845 & AY292966 \\
\hline Vibrio fischeri SI1D & Sepiola intermedia & France (Banyuls sur Mer) & AY292948 & AY292961 & AY292984 \\
\hline Vibrio fischeri SL518 & Sepiola ligulata & France (Banyuls sur Mer) & AY292950 & AY292962 & AY292985 \\
\hline Vibrio fischeri SR5 & Sepiola robusta & France (Banyuls sur Mer) & AY292926 & AF034851 & AY292972 \\
\hline Vibrio fischeri WH1 & Free-living & Massachusetts, USA (Woods Hole) & AY292930 & AY292955 & AY292973 \\
\hline Vibrio fluvialis & NCTC $11327^{\mathrm{T}}$ & & X76335 & & \\
\hline Vibrio furnissii & ATCC $35016^{\mathrm{T}}$ & & $\mathrm{X} 74704$ & & \\
\hline Vibrio gazogenes & ATCC $29988^{\mathrm{T}}$ & & X74705 & & \\
\hline Vibrio harveyi & ATCC $14126^{\mathrm{T}}$ & & X56578 & & \\
\hline Vibrio hollisae & ATCC $33564^{\mathrm{T}}$ & & X56583.1 & & \\
\hline Vibrio iliopiscarius & ATCC $51760^{\mathrm{T}}$ & & AB000278 & & \\
\hline Vibrio lentus Sat101 & Sepiola atlantica & Spain (Vigo) & AY292935 & & \\
\hline Vibrio lentus Sat201 & Sepiola atlantica & Spain (Vigo) & AY292936 & & \\
\hline Vibrio lentus SI01 & Sepiola intermedia & France (Banyuls sur Mer) & AY292927 & & \\
\hline Vibrio logei & AHL vs15 & & AY292931 & & \\
\hline Vibrio logei & ATCC 15382 & & AY292932 & AY292956 & AY292974 \\
\hline Vibrio logei & ATCC 35077 & & AY292933 & & \\
\hline Vibrio logei SA112 & Sepiola affinis & France (Banyuls sur Mer) & AY292925 & & \\
\hline Vibrio logei SI1E & Sepiola intermedia & France (Banyuls sur Mer) & AY292949 & & \\
\hline Vibrio logei SL101 & Sepiola ligulata & France (Banyuls sur Mer) & AY292928 & & \\
\hline
\end{tabular}


Table 1. cont.

\begin{tabular}{|c|c|c|c|c|c|}
\hline Strain & Source & Location & 16S rRNA & $\operatorname{gapA}$ & $\operatorname{luxRI}$ \\
\hline Vibrio logei SR181 & Sepiola robusta & France (Banyuls sur Mer) & AY292934 & AY292957 & AY292975 \\
\hline Vibrio logei Srond 101401 & Sepiola rondeleti & France (Banyuls sur Mer) & AY292929 & & \\
\hline Vibrio marinus & NCIMB $1144^{\mathrm{T}}$ & & X82142 & & \\
\hline Vibrio mediterranei & CIP $103203^{T}$ & & X74710 & & \\
\hline Vibrio metschnikovii & CIP $69.14^{\mathrm{T}}$ & & X74711 & & \\
\hline Vibrio mimicus & ATCC $33653^{\mathrm{T}}$ & & AY292937 & & AY292976 \\
\hline Vibrio mytili & & & X99761 & & \\
\hline Vibrio natriegens & ATCC $14048^{\mathrm{T}}$ & & X56581 & & \\
\hline Vibrio navarrensis & CIP $103381^{\mathrm{T}}$ & & X74715 & & \\
\hline Vibrio nereis & ATCC $25917^{\mathrm{T}}$ & & $\mathrm{X} 74716$ & & \\
\hline Vibrio nigripulchritudo & ATCC $27043^{\mathrm{T}}$ & & X74717 & & \\
\hline Vibrio ordalii & ATCC $33509^{\mathrm{T}}$ & & X74718 & & \\
\hline Vibrio orientalis & ATCC $33934^{\mathrm{T}}$ & & X74719 & & \\
\hline Vibrio parahaemolyticus & ATCC $17802^{\mathrm{T}}$ & & AY303756 & & AY292965 \\
\hline Vibrio pectenicida & Ifremer $\mathrm{A} 365^{\mathrm{T}}$ & & Y13830 & & \\
\hline Vibrio proteolyticus & ATCC $15338^{\mathrm{T}}$ & & X56579 & & \\
\hline Vibrio salmonicida & Salmo salar & & AY292918 & & \\
\hline Vibrio scophthalmi & Scophthalmus maximus & & U46579 & & \\
\hline Vibrio shiloi & $\mathrm{AK} 1^{\mathrm{T}}$ & & AF007115 & & \\
\hline Vibrio splendidus & KAT16 & & AF025329 & & \\
\hline Vibrio tapetis & CECT $4600^{\mathrm{T}}$ & & Y08430 & & \\
\hline Vibrio tubiashii & ATCC $19109^{\mathrm{T}}$ & & $\mathrm{X} 74725$ & & \\
\hline Vibrio vulnificus & C7184 & & X76334 & & \\
\hline
\end{tabular}

sequence alignment (Wheeler, 1998). The results of this procedure are directly compatible with parsimony-based tree lengths and the method appears to generate more efficient (and therefore simpler) explanations of sequence variation than multiple sequence alignment (Wheeler, 1996). Direct optimization, although computationally intense, is much less demanding than parsimony-based multiple sequence alignment algorithms. The method has also been demonstrated to yield more congruent results than multiple sequence alignments when congruence among partitions is used as a criterion (Wheeler \& Hayashi, 1998).

Character transformations were weighted differentially to observe how they affect phylogenetic conclusions [sensitivity analysis sensu Wheeler (1995)]. A parameter space of two analytical variables was examined: insertion-deletion cost ratio and transversion-transition cost ratio. When the transversion-transition ratio was set at a value other than unity, the insertion-deletion cost was set according to the cost of transversions. In total, 20 combinations of parameters were employed in the analysis [insertion-deletion ratios of 1,2, 4 and 8; transversion-transition ratios of $1,2,4,8$ and infinite (transversion parsimony)]. This method allows discernment between stable (those supported by analysis using a wide range of parameters) and unstable (those that only appear under particular parameter sets) relationships.

Molecular data analysis. The three molecular partitions were analysed independently and combined directly, with all characters weighted equally without regard to source. These datasets are referred as 16S (16S rRNA dataset alone), gapA (gapA dataset alone), luxRI (luxRI dataset alone) and molecular $(16 \mathrm{~S}+$ gapA+ luxRI). The tree-search strategy adopted combined SPR (subtree pruning and regrafting) and TBR (tree bisection and reconnection) branch-swapping on the best of 50 random-addition replicates (-random, 50), holding 100 trees per round (-maxtrees, 100) and performing one round of tree-fusing (Goloboff, 1999). The commands '-slop 5' and '-checkslop 10' were used; these commands are intended to check all cladogram lengths that are within $n$-tenths of a percentage of the current minimum value. A slop value of 10 would check all cladograms found within $1 \%$ of the minimum tree length. This option slows down the search, but is less affected by the heuristics of the tree-length calculation shortcuts. The 'implied alignments' obtained with POY were checked with PAUP* $4 \mathrm{~b}$ (Swofford, 2002) and identical tree lengths were obtained. These implied alignments were used to compute jackknife values by performing 1000 randomaddition replicates.

Biochemical and restriction digest data analysis. Parsimony analysis of the biochemical/restriction digest dataset was performed with POY. The tree-search strategy adopted involved a heuristic algorithm with random-addition sequence and TBR branch-swapping, similar to the methods described for the molecular data analysis (Wheeler et al., 2002).

Combined analysis. Morphological and total molecular data were combined directly and analysed by using direct optimization for the same 20 parameter sets that were applied to each one of the individual molecular partitions and to the combined molecular analysis. Morphological transformations were weighted equally to the highest of the molecular costs (indels), to diminish the potentially overwhelming effect of molecular data versus morphology. Bremer support values were estimated by using the heuristics procedure implemented in POY (-bremer -constrain 'filename' -topology 'treetopology-in-parenthetical-notation'). In total, we analysed seven partitions and 20 parameter sets per partition (140 analyses) that were executed in a $866 \mathrm{MHz}$ Pentium III processor (RDRAM, $256 \mathrm{Mb}$ ). 


\section{RESULTS AND DISCUSSION}

The use of several loci, combined with biochemical and RFLP attributes among Vibrio taxa, is a step forward in understanding the relationships among members of the group. Alternatives to the use of all available evidence must explicitly exclude sets of the data (Kluge, 1989; Giribet et al., 2001). Once any set of characters has been excluded, a bias towards those characters included in the analysis will be evident. Combining all the evidence available allows a phylogenetic framework to be formulated with the maximum explanatory power to produce the least biased result. In this respect, our analysis constitutes the first totalevidence analysis to study bacterial relationships, a common practice in systematic zoology and botany (Giribet, 2002; Giribet \& Wheeler, 2002).

Phylogenetic analysis using a combined approach that searches for the most parsimonious solution for character variation appears to be a new method that can be applied not only to metazoan phylogenies, but also to prokaryotic systematics. Molecular data were analysed by using the direct optimization procedure, which derives cladogram costs without multiple sequence alignment. This methodology accommodates sequence length variation as transformations that involve the addition, deletion and substitution of nucleotides, as opposed to the addition of unobservable 'gaps'. Direct optimization produces more parsimonious and more congruent results than multiple sequence alignment in a shorter amount of time (Giribet et al., 2001). POY minimizes the weighted number of evolutionary changes over the entire tree, working in a onestep fashion as opposed to the more classical two-step analyses (alignment and tree search). Nodal support was evaluated by the jackknifing method with a deletion ratio of $e^{-1}$ (about $0 \cdot 36$ ), in which every character has the same chance of being omitted from a given pseudoreplicate (Farris, 1970). Therefore, the single phylogeny reported here represents the most parsimonious tree with the least amount of bias.

The cladogram from the combined evidence of biochemical characters and molecular data, which minimizes overall incongruence among data partitions (utilizing character congruence as a meta-optimality criterion) (Wheeler, 1995), demonstrates the non-monophyly of the Vibrionaceae (Fig. 1). Several clades contain symbiotic vibrios, irrespective of the type of host (i.e. mollusc, annelid, crustacean or fish) or geographical area where they were found (Table 1). This provides evidence that symbiosis has arisen multiple times within the family Vibrionaceae, although support for several nodes is below the commonly used $50 \%$ threshold. Also, some free-living species (V. fischeri ATCC $7744^{\mathrm{T}}$ and Photobacterium leiognathi) that were included in the analyses were not monophyletic, whereas $V$. fischeri clade A, which includes both free-living and symbiotic isolates, is supported by the analyses ( $95 \%$ jackknife support). Two other symbiotic strains isolated from sepiolid squids (EH201 and EH701) did not group with the clades that contained the remainder of squid host strains (clades A, B and C; Fig. 1). It is important to note that not all squid symbionts in these clades are from the same geographical location (Indo-west Pacific); in fact, several sister groups are constituted by symbiotic isolates from different host squids (Table 1). Whether V. fischeri ATCC $7744^{\mathrm{T}}$ is an

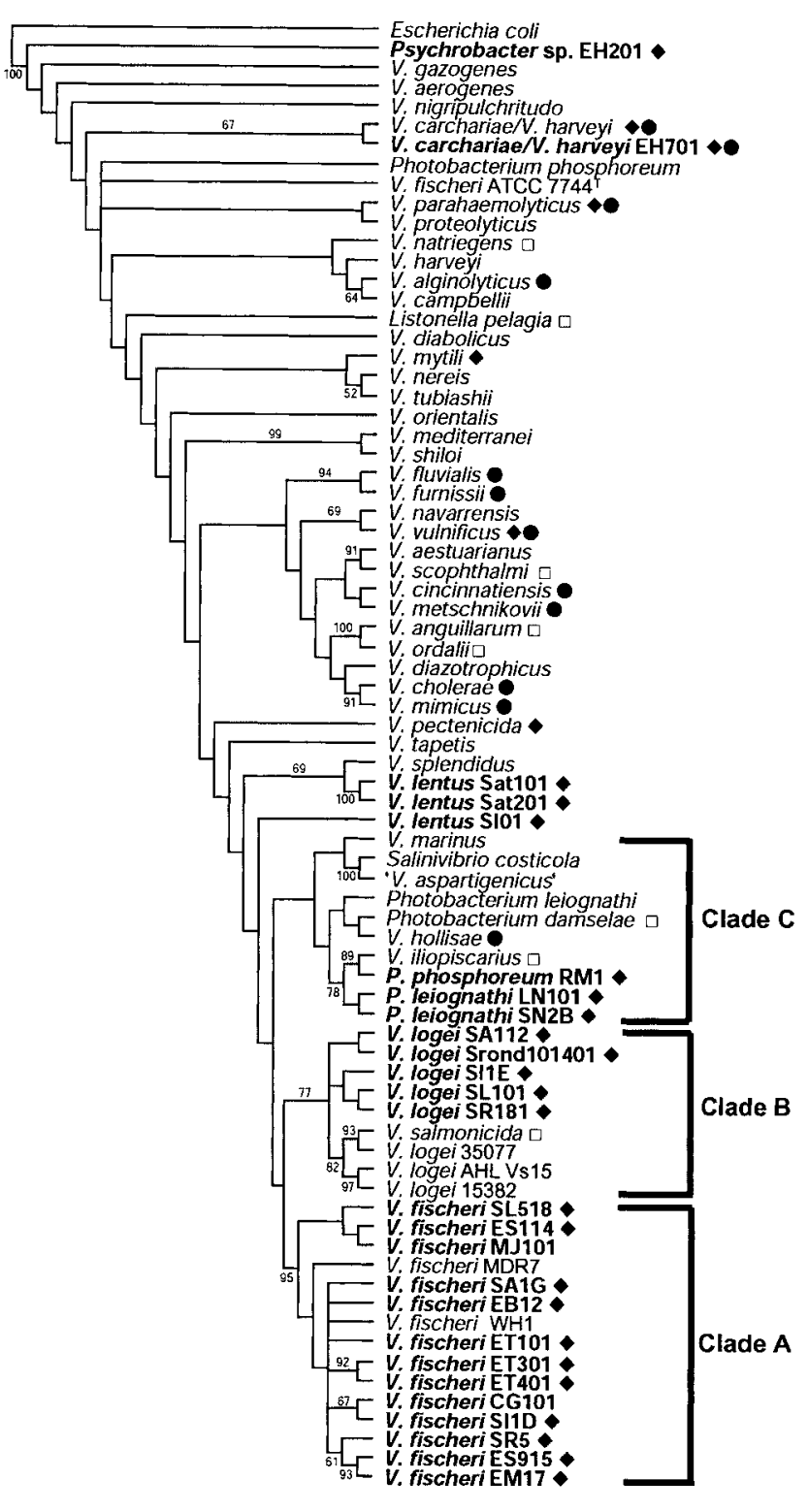

Fig. 1. Phylogenetic tree of Vibrionaceae lineages, based on the DNA sequences of three loci and 100 biochemical characters for the parameter set that minimizes overall incongruence among the four partitions. The single tree was obtained when all insertions and deletions equalled all other genetic changes (transitions/transversions). Jackknife values $>50 \%$ are shown at nodes. Taxa that have symbiotic relationships with molluscan hosts; $\square$, fish pathogens; $\bullet$, taxa that are human pathogens in the environment. All light organ symbionts (fish or squid) are shown in bold. 
ancestral Vibrio biotype that has remained separate from other $V$. fischeri environmental isolates because it is in culture remains to be determined. Future investigations should include not only strain types that have been used in previous studies (i.e. V. fischeri ATCC $7744^{\mathrm{T}}$ ), but also those that have recently been isolated from environmental samples, which may represent the current population of vibrios that are more likely to share a common or closely related ancestral lineage.

Vibrio logei isolates (both free-living and symbiotic) used in the analyses were found to be polyphyletic, with one clade (B) clustering with the $V$. fischeri clade of symbiotic strains (clade A). Clade B contains several isolates of $V$. logei (both symbiotic and free-living) and Vibrio salmonicida (a salmon pathogen), whereas a third, smaller Vibrio lentus clade is sister to Vibrio splendidus (69\% jackknife support). The finding of a well-supported clade ( $82 \%$ jackknife frequency) that contains V. logei strains ATCC 35077, AHL Vs15 and SR181 and Vibrio salmonicida suggests that V. salmonicida might have evolved from a free-living $V$. logei congener (Wiik et al., 1995). Embedded within the large clade that contains V. logei and V. fischeri are several strains of Photobacterium leiognathi, Photobacterium phosphoreum and Photobacterium damselae (clade C; Fig. 1), which supports previous data that suggested that Photobacterium and Vibrio may be monophyletic (MacDonell \& Colwell, 1985; Ruimy et al., 1994). Nesting of Photobacterium within the genus Vibrio implies that our phylogeny supports this hypothesis, but it also includes other taxa besides these two genera. More data are needed on the inclusion of other hypothesized sister taxa, which may help to further define this group of luminous bacteria.

Although much of the species relatedness differs from that reported in previous phylogenetic studies (Bryant et al., 1986; Dorsch et al., 1992; Kita-Tsukamoto et al., 1993; Alsina \& Blanch, 1994; Martin-Kearley \& Gow, 1994; Urakawa et al., 1997, 1999), several robust clades are apparent in our phylogeny that have been supported by other studies. Salinivibrio costicola, $P$. damselae and $P$. leiognathi have previously been proposed to be sister to one another (Kita-Tsukamoto et al., 1993; Alsina \& Blanch, 1994; Ruimy et al., 1994); our results support this hypothesis, but also include other Vibrio species that do not cluster with S. costicola (Fig. 1). Another relationship supported by our phylogeny is that of human pathogens Vibrio fluvialis, Vibrio furnissii, Vibrio cincinnatiensis, Vibrio metschnikovii, $V$. cholerae and Vibrio mimicus, which are known to cause enteric distress (Daniels \& Shafaie, 2000). Sister group relationships among these pathogenic species have high support values ( $V$. fluvialis/V. furnissii, 94\%; Vibrio anguillarum/Vibrio ordalii, $100 \%$; V. cholerae/V. mimicus, $91 \%)$ and are most likely to have arisen from a virulent strain. Again, strict monophyly among pathogenic strains is not supported in this clade. Other symbiotic species, such as the $V$. fischeri- $V$. logei- $V$. salmonicida clade, are also supported in our analyses, as in studies that used $5 \mathrm{~S}$
rRNA (Ruimy et al., 1994) and partial 16S rRNA (KitaTsukamoto et al., 1993) gene sequences. Finally, the alliance between $V$. cholerae and $V$. mimicus was well-supported (91\% jackknife frequency), which was also shown by using 16S rRNA (Kita-Tsukamoto et al., 1993) and non-sequence characters (Davis et al., 1981). From this study and others, $V$. mimicus is classified as an atypical strain of $V$. cholerae and probably contains a reduced genome component from its ancestral parent. This was apparent as the $16 \mathrm{~S}$ rRNA gene sequence was substantially smaller than those of all other vibrios sequenced in this study.

Of importance to this study are the multiple origins of pathogenic/symbiotic strains of Vibrio and related genera. As the association between vibrios and invertebrates existed before pathogenic Vibrio-human interactions, it appears that tissue-colonization mechanisms were first utilized by mutualists and were then enlisted for use in pathogenic associations (Ruby, 1999a; Schjørring \& Koella, 2003). Unfortunately, very little is known about communication, regulation or co-evolution among interactive species (Nishiguchi, 2001). It is relatively unknown how hostmutualist relationships are disrupted and/or capitalized by pathogenic bacteria (Ruby, 1999b). Evolutionary transitions between mutualism and pathogenicity would produce a mosaic of types of interactions within the tree, resulting in close relationships between the two (Moran \& Wernegreen, 2000), as suggested by our data. Our phylogenetic tree of Vibrio species is the first to combine molecular and biochemical information for this important group of bacteria and provides new insight on the evolution of both types of association. Future work should include a search for virulence/pathogenicity islands in symbiotic organisms within the genus Vibrio, which may help us to further our knowledge of the evolution of pathogenesis. If heterozygous islands are found in nonpathogenic species of Vibrio, they can then be compared to those within the genomes of pathogenic Vibrio species (Makino et al., 2003). Similarities or differences between the two would lead to further insight in the development of pathogenicity in benign organisms.

\section{ACKNOWLEDGEMENTS}

The authors would like to thank members of the Nishiguchi lab and two anonymous referees for help with reviewing the manuscript and other forms of advice during data collection. We would especially like to thank Charity Licklite and Melanie Sanchez for their assistance in sequencing bacterial loci. Funding was provided by NSF DBI-0079820, NSF SBE-0123690 and NIH SO6-GM08136-26 to M. K. N. and grants NIH-RISE GM61222-01 and NIH-MARC GM07667-24 at NMSU for support of minority undergraduates who helped with this project.

\section{REFERENCES}

Alsina, M. \& Blanch, A. R. (1994). Improvement and update of a set of keys for biochemical identification of Vibrio species. J Appl Bacteriol 77, 719-721. 
Bang, S. S., Baumann, L., Woolkalis, M. J. \& Baumann, P. (1981). Evolutionary relationships in Vibrio and Photobacterium as determined by immunological studies of superoxide dismutase. Arch Microbiol 130, 111-120.

Baumann, P., Baumann, L. M., Woolkalis, J. \& Bang, S. S. (1983). Evolutionary relationships in Vibrio and Photobacterium: a basis for a natural classification. Annu Rev Microbiol 37, 369-398.

Bryant, T. N., Lee, J. V., West, P. A. \& Colwell, R. R. (1986). Numerical classification of species of Vibrio and related genera. J Appl Bacteriol 61, 437-467.

Czyż, A., Wróbel, B. \& Węgrzyn, G. (2000). Vibrio harveyi bioluminescence plays a role in stimulation of DNA repair. Microbiology 146, 283-288.

Daniels, N. A. \& Shafaie, A. (2000). A review of pathogenic Vibrio infections for clinicians. Infect Med 17, 665-685.

Davis, B. R., Fanning, G. R., Madden, J. M., Steigerwalt, A. G., Bradford, H. B., Jr, Smith, H. L., Jr \& Brenner, D. J. (1981). Characterization of biochemically atypical Vibrio cholerae strains and designation of a new pathogenic species, Vibrio mimicus. J Clin Microbiol 14, 631-639.

de Pinna, M. C. C. (1991). Concepts and tests of homology in the cladistic paradigm. Cladistics 7, 367-394.

Dorsch, M., Lane, D. \& Stackebrandt, E. (1992). Towards a phylogeny of the genus Vibrio based on 16S rRNA sequences. Int J Syst Bacteriol 42, 58-63.

Farris, J. S. (1970). Methods for computing Wagner trees. Syst Zool 19, 83-92.

Giribet, G. (2002). Relationships among metazoan phyla as inferred from $18 \mathrm{~S}$ rRNA sequence data: a methodological approach. In Molecular Systematics and Evolution: Theory and Practice, pp. 85-101. Edited by R. DeSalle, G. Giribet \& W. C. Wheeler. Basel, Switzerland: Birkhäuser.

Giribet, G. \& Wheeler, W. C. (2002). On bivalve phylogeny: a high-level analysis of the Bivalvia (Mollusca) based on combined morphology and DNA sequence data. Invertebr Biol 121, 271-324.

Giribet, G., Edgecombe, G. D. \& Wheeler, W. C. (2001). Arthropod phylogeny based on eight molecular loci and morphology. Nature 413, 157-161.

Goloboff, P. A. (1999). Analyzing large data sets in reasonable times: solutions for composite optima. Cladistics 15, 415-428.

Kita-Tsukamoto, K., Oyaizu, H., Nanba, K. \& Simidu, U. (1993). Phylogenetic relationships of marine bacteria, mainly members of the family Vibrionaceae, determined on the basis of 16S rRNA sequences. Int J Syst Bacteriol 43, 8-19.

Kluge, A. G. (1989). A concern for evidence and a phylogenetic hypothesis of relationships among Epicrates (Boidae, Serpentes). Syst Zool 38, 7-25.

Koropatnick, T., Estrada, A., Apicella, M. A. \& McFall-Ngai, M. J. (2001). Messengers or mercenaries? Characterizing the role of macrophage-like hemocytes in the bacteria-induced morphogenesis of the juvenile light organ of Euprymna scolopes. Am Zool 41, 1496 (abstract no. P1.24).

MacDonell, M. T. \& Colwell, R. R. (1985). Phylogeny of the Vibrionaceae, and recommendation for two new genera, Listonella and Shewanella. Syst Appl Microbiol 6, 171-182.

Maddison, D. R. \& Maddison, W. P. (2002). MacClade. Sunderland, MD: Sinauer Associates.

Makino, K., Oshima, K., Kurokawa, K. \& 14 other authors (2003). Genome sequence of Vibrio parahaemolyticus: a pathogenic mechanism distinct from that of $V$. cholerae. Lancet 361, 743-749.
Martin-Kearley, J. \& Gow, J. A. (1994). Numerical taxonomy of Vibrionaceae from Newfoundland coastal waters. Can J Microbiol 40, 355-361.

McFall-Ngai, M. J. (1999). Consequences of evolving with bacterial symbionts: lessons from the squid-vibrio association. Annu Rev Ecol Syst 30, 235-256.

Moran, N. A. \& Wernegreen, J. J. (2000). Lifestyle evolution in symbiotic bacteria: insights from genomics. Trends Ecol Evol 15, 321-326.

Nearhos, S. P. \& Fuerst, J. A. (1987). Reanalysis of 5S rRNA sequence data for the Vibrionaceae with the Clustan Program Suite. Curr Microbiol 15, 329-335.

Nishiguchi, M. K. (2000). Temperature affects species distribution in symbiotic populations of Vibrio spp. Appl Environ Microbiol 66, 3550-3555.

Nishiguchi, M. K. (2001). Co-evolution of symbionts and hosts: the sepiolid-Vibrio model. In Symbiosis: Mechanisms and Model Systems, pp. 757-774. Edited by J. Seckbach. Dordrecht, the Netherlands: Kluwer.

Nishiguchi, M. K. (2002). Host-symbiont recognition in the environmentally transmitted sepiolid squid-Vibrio mutualism. Microb Ecol 44, 10-18.

Nishiguchi, M. K. \& Jones, B. W. (2003). Microbial biodiversity within the Vibrionaceae. In Origins, Evolution, and the Biodiversity of Microbial Life. Edited by J. Seckback. Dordrecht, the Netherlands: Kluwer (in press).

Nishiguchi, M. K., Ruby, E. G. \& McFall-Ngai, M. J. (1998). Competitive dominance among strains of luminous bacteria provides an unusual form of evidence for parallel evolution in sepiolid squid-vibrio symbioses. Appl Environ Microbiol 64, 3209-3213.

Nyholm, S. V., Stabb, E. V., Ruby, E. G. \& McFall-Ngai, M. J. (2000). Establishment of an animal-bacterial association: recruiting symbiotic vibrios from the environment. Proc Natl Acad Sci U S A 97, 10231-10235.

Reich, K. A. \& Schoolnik, G. K. (1994). The light organ symbiont Vibrio fischeri possesses a homolog of the Vibrio cholerae transmembrane transcriptional activator ToxR. J Bacteriol 176, 3085-3088.

Reich, K. A. \& Schoolnik, G. K. (1996). Halovibrin, secreted from the light organ symbiont Vibrio fischeri, is a member of a new class of ADP-ribosyltransferases. J Bacteriol 178, 209-215.

Reich, K. A., Biegel, T. \& Schoolnik, G. K. (1997). The light organ symbiont Vibrio fischeri possesses two distinct secreted ADPribosyltransferases. J Bacteriol 179, 1591-1597.

Ruby, E. G. (1999a). Ecology of a benign "infection": colonization of the squid luminous organ by Vibrio fischeri. In Microbial Ecology and Infectious Disease, pp. 217-231. Edited by E. Rosenberg. Washington, DC: American Society for Microbiology.

Ruby, E. G. (1999b). The Euprymna scolopes-Vibrio fischeri symbiosis: a biomedical model for the study of bacterial colonization of animal tissue. J Mol Microbiol Biotechnol 1, 13-21.

Ruby, E. G. \& Asato, L. M. (1993). Growth and flagellation of Vibrio fischeri during initiation of the sepiolid squid light organ symbiosis. Arch Microbiol 159, 160-167.

Ruby, E. G. \& Lee, K.-H. (1998). The Vibrio fischeri-Euprymna scolopes light organ association: current ecological paradigms. Appl Environ Microbiol 64, 805-812.

Ruimy, R., Breittmayer, V., Elbaze, P., Lafay, B., Boussemart, O., Gauthier, M. \& Christen, R. (1994). Phylogenetic analysis and assessment of the genera Vibrio, Photobacterium, Aeromonas, and Plesiomonas deduced from small-subunit rRNA sequences. Int J Syst Bacteriol 44, 416-426. 
Schjørring, S. \& Koella, J. C. (2003). Sub-lethal effects of pathogens can lead to the evolution of lower virulence in multiple infections. Proc R Soc Lond B Biol Sci 270, 189-193.

Stabb, E. V. \& Ruby, E. G. (2003). Contribution of pilA to competitive colonization of the squid Euprymna scolopes by Vibrio fischeri. Appl Environ Microbiol 69, 820-826.

Stabb, E. V., Reich, K. A. \& Ruby, E. G. (2001). Vibrio fischeri genes $h v n A$ and $h v n B$ encode secreted $\mathrm{NAD}^{+}$-glycohydrolases. J Bacteriol 183, 309-317.

Stewart, J. J., Nyholm, S. V. \& McFall-Ngai, M. J. (2001). Squid hemocytes are able to discriminate the specific symbiont from other types of bacteria. Am Zool 41, 1595-1596 (abstract no. 62.3).

Swofford, D. L. (2002). PAUP*: Phylogenetic Analysis Using Parsimony ( ${ }^{*}$ and other methods). Sunderland, MA: Sinauer Associates.

Urakawa, H., Kita-Tsukamoto, K. \& Ohwada, K. (1997). 16S rDNA genotyping using PCR/RFLP (restriction fragment length polymorphism) analysis among the family Vibrionaceae. FEMS Microbiol Lett 152, 125-132.

Urakawa, H., Kita-Tsukamoto, K. \& Ohwada, K. (1999). Restriction fragment length polymorphism analysis of psychrophilic and psychrotrophic Vibrio and Photobacterium from the north-western Pacific Ocean and Otsuchi Bay, Japan. Can J Microbiol 45, 67-76.

Wheeler, W. C. (1995). Sequence alignment, parameter sensitivity, and the phylogenetic analysis of molecular data. Syst Biol 44, 321-331.

Wheeler, W. C. (1996). Optimization alignment: the end of multiple sequence alignment in phylogenetics? Cladistics 12, 1-9.

Wheeler, W. C. (1998). Alignment characters, dynamic programming and heuristic solutions. In Molecular Approaches to Ecology and Evolution, vol. 1, pp. 243-251. Edited by R. DeSalle \& B. Schierwater. Basel, Switzerland: Birkhäuser Verlag.

Wheeler, W. C. \& Hayashi, C. Y. (1998). The phylogeny of extant chelicerate orders. Cladistics 14, 173-192.

Wheeler, W. C., Gladstein, D. S. \& DeLaet, J. (2002). POY: the optimization of alignment characters. New York: American Museum of Natural History.

Wiik, R., Stackebrandt, E., Valle, O., Daae, F. L., Rødseth, O. M. \& Andersen, K. (1995). Classification of fish-pathogenic vibrios based on comparative 16S rRNA analysis. Int J Syst Bacteriol 45, 421-428. 